SHORT REPORT

\title{
Autoantibodies to glutamic acid decarboxylase in downbeat nystagmus
}

\author{
G Antonini, R Nemni, F Giubilei, F Gragnani, V Ceschin, S Morino, E Bucci, \\ N Accornero
}

J Neurol Neurosurg Psychiatry 2003;74:998-999

The cause of downbeat nystagmus (DBN) remains undiagnosed in about $40 \%$ of patients. This paper reports the presence of antiglutamic acid decarboxylase antibodies (GAD-Ab) in a patient with DBN. Antibodies against GABAergic neurons located in the vestibular complex may induce chemical denervation of the floccular neurons, which normally suppress the peripheral imbalance between vertical semicircular canal systems, thereby causing DBN. Testing for GAD-Ab may be indicated in DBN patients without an identifiable anatomical brain lesion.

$\mathrm{D}$ ownbeat nystagmus (DBN) is defined as a spontaneous nystagmus present with fixation in the primary position or on lateral gaze, characterised by downward fast phases. A proportion of patients with DBN have an identifiable anatomical lesion of the vestibulocerebellum and underlying medulla. However, the cause of DBN remains undiagnosed in about $40 \%$ of patients, in whom nystagmus is considered idiopathic.

Glutamic acid decarboxylase (GAD) is the enzyme that catalyses the conversion of glutamic acid to $\gamma$-aminobutyric acid (GABA). GAD is mainly expressed in pancreatic $\beta$ cells and GABA secreting neurons, which are widely represented in the central nervous system. Other than in insulin dependent diabetes mellitus (IDDM) and autoimmune polyendocrine syndrome, GAD has also been found to be a target antigen of humoral autoimmunity in some neurological disorders. ${ }^{2}$ We report a patient with DBN in association with a high titre of anti-GAD antibodies (GAD-Ab).

\section{PATIENT HISTORY}

A 66 year old woman was admitted to our hospital in June 2000 with a three month history of progressive oscillopsia, blurred vision, and vertigo. The neurological examination showed spontaneous vertical DBN in the primary position and on lateral gaze, not inhibited by fixation, and unsteadiness with lateral propulsion to the right side. Convergence did not influence DBN. Coordination, muscle strength, deep tendon reflexes, and sensory examination were normal. She did not have painful muscle cramps or stiffness either at rest or during voluntary activity. No palatal myoclonus was detected.

Routine laboratory tests showed erythorcyte sedimentation rate $30 \mathrm{~mm}$ in 1st h. Neoplastic antigens (CEA, Ca 125, Ca 19-9, NSE, TPA, $\alpha$-fetoprotein, ferritin) were normal. Serological tests showed a GAD-Ab titre $>100 \mathrm{U} / \mathrm{ml}$, as well as positive anti-islet cell antigen and antiparietal cell antigen antibody. Other organ specific antibodies tested (anti-Yo, anti-Hu, ANA, ENA, ANCA, and ACLA) were normal.

The CSF examination revealed the presence of oligoclonal bands and GAD-Ab (titre $>100 \mathrm{U} / \mathrm{ml}$ ). Brain magnetic resonance imaging and angio magnetic resonance imaging, total body computed tomography, mammography, oesophagogastroduodenoscopy, pelvic echography, colposcopy, as well as EMG and nerve conduction studies were normal. Immunological (corticosteroid, immunoglobulin, and plasmapheresis) and symptomatic (clobazam and gabapentin) treatments were performed in the subsequent months. Oscillopsia, blurred vision, and vertigo progressively worsened until, after eight months, the patient was unable to walk without help. The neurological examination, confirmed DBN and severe unsteadiness with lateral propulsion. High GAD-Ab titre $(>100 \mathrm{U} / \mathrm{ml})$ in both serum and CSF was confirmed. A further complete oncological follow up and brain magnetic resonance imaging were normal. After a two year follow up DBN and unsteadiness were unchanged. A last oncological follow up performed in May 2002 was normal.

\section{IMMUNOLOGICAL STUDY}

GAD-Abs were detected by immunohistochemistry and immunoprecipitation. Immunostaining of a frozen section of rat cerebellum with patient serum showed positive staining in nerve terminals at the base of Purkinje cells and in the glomeruli of the granular layer with a typical GAD-Ab pattern. GAD-Ab reactivity was confirmed in an immunoprecipitation assay of ${ }^{35} \mathrm{~S}$-methionine labelled, in vitro translated, full length recombinant human $\mathrm{GAD}_{65}$. Forty four blood donors, one patient with palatal myoclonus, five with stiff man syndrome, one with subacute cerebellar ataxia, and 19 with IDDM were used as controls. GAD-Ab titres in DBN patients were high $(>100 \mathrm{U} / \mathrm{ml})$ in both serum and CSF, and similar to those found in patients with stiff man syndrome and palatal myoclonus. The GAD-Ab titre in the serum of the patient with cerebellar ataxia was $42 \mathrm{U} / \mathrm{ml}$ and ranged from 1 to $14 \mathrm{U} / \mathrm{ml}$ in the IDDM patients. Control serum samples from blood donors were negative.

To characterise the GAD antibody reactivity, fragments (amino acids 290-585 or amino acids 290-511) of the full length (amino acids 1-585) recombinant human GAD65 were produced and tested with serum samples from our patient with DBN, as well as from patients with palatal myoclonus, stiff man syndrome, cerebellar ataxia, and IDDM. Strong reactivity (>100 U/ml) against the GAD fragments 290-585 was present in serum from DBN, as well as in sera from patients with palatal myoclonus and stiff man syndrome. Sera from the patient with cerebellar ataxia and from all but one of the IDDM patients tested negative. Reactivity against the GAD 290-511 was negative in all sera.

Abbreviations: DBN, downbeat nystagmus; GAD-Ab, antiglutamic decarboxylase antibodies; GABA, $\gamma$-aminobutyric acid; IDDM, insulin dependent diabetes mellitus 


\section{DISCUSSION}

Autoantibodies that target the two isoforms of GAD (GAD 65 and GAD 67) are increased in a high proportion of patients with stiff man syndrome ${ }^{2-4}$ as well as in those with progressive encephalomyelitis with rigidity and myoclonus. ${ }^{2}$ They have, however, also been reported in patients with other neurological diseases. ${ }^{2}$ Subacute cerebellar ataxia, ${ }^{5}$ epilepsy, ${ }^{6}$ and palatal myoclonus ${ }^{7}$ have all been reported within the spectrum of $\mathrm{GAD}-\mathrm{Ab}$ associated neurological syndromes over the past decade. To our knowledge, no cases of DBN associated with GAD-Ab have previously been reported. Different GABAergic pathways are involved in GAD-Ab associated neurological syndromes, which may explain why GAD-Ab acts on specific anatomical structures. For example, GAD-Ab in the CSF of an ataxic patient selectively suppressed GABA mediated transmission on cerebellar Purkinje cells by acting on the presynaptic terminals of GABAergic interneurons and decreasing GABA release on Purkinje cells. ${ }^{8}$ In stiff man syndrome, an action of GAD-Abs on GABAergic neurons controlling motor unit activity in the spinal cord has been hypothesised.

Usually, DBN is of central origin and is associated with lesions of the vestibulocerebellum and underlying medulla. ${ }^{9}$ Vertical vestibulo-ocular reflexes are mainly governed by the vertical semicircular canal system. While right and left horizontal semicircular canal system activities balance each other, the activity of the anterior canal (responsible for upward eye movement) on one side must be balanced by the activity of the posterior canal (responsible for downward eye movement) on the other side..$^{10}$ The cerebellar flocculus inhibits anterior canal pathways though not the posterior canal pathways. ${ }^{11}$ Consequently, experimental lesions to the vestibulocerebellum (flocculectomy in the monkey) remove inhibition from the anterior, though not posterior canal projections, and produce DBN. ${ }^{12}$ A GABAergic transmission from Purkinje cells to floccular neurons has been suggested. ${ }^{13}$ It is possible that a primary action of GAD-Abs against GABAergic neurons induces chemical denervation of the floccular neurons, thereby causing DBN.

The heterogeneity of GAD-Ab syndromes may be related to different antigenic targets. First of all, it should be borne in mind that different GAD molecules (GAD65, GAD67), expressed in the various cells in the central nervous system, might constitute a variable element in the immunopathogenesis of the aforementioned neurological syndromes. Furthermore, immunological studies have shown considerable heterogeneity between the anti-GAD responses in stiff man syndrome and those in IDDM: higher autoantibody levels and recognition of a linear terminal epitope in the autoantigen GAD-65 distinguish stiff man syndrome from IDDM.${ }^{14}$ Finally, on western blots, GAD-Abs recognise GAD in stiff man syndrome, though not in IDDM, which indicates that antibody reactivity in IDDM is aimed at a conformational epitope rather than a linear epitope. ${ }^{15}$ Our study reveals some important immunological similarities in stiff man syndrome, palatal myoclonus and $\mathrm{DBN}$ as regards GAD-Ab titre and reactivity: antibody titres were high and antibodies bound epitopes contained in the terminal portion of GAD-65. The serological findings in cerebellar ataxia appeared more similar to those in IDDM: GAD-Abs were less reactive and target epitopes were crucially dependent upon the molecule being intact (Nemni 1996, personal communication). These findings imply that, though different neurological and non-neurological syndromes may show a macroscopically similar antibody response to GAD, the fine details of this response might characterise different phenotypic expression of the syndromes. The positive CSF oligoclonal bands in our patient may suggest a chronic immunological response directed against neural antigens. As oligoclonal bands represent IgGs with their own specificity, we can hypothesise that they may be directed against different epitopes on the same antigen, as it possibly happens in a percentage of patients with stiff man syndrome and IDDM. Nevertheless, to date we have no data indicating an involvement of different epitopes in anti-GAD associated neurological syndromes. We may also hypothesise that a focal increased permeability in the blood-brain barrier (for example, after vasculitis or encephalitis) might permit or even increase antibody access to restricted areas of the central nervous system, though both the lack of magnetic resonance imaging evidence of brain lesions and the normality of the organ specific antibodies tested lead us to exclude a vasculitic process in the patient described in this study.

In conclusion, other than stiff man syndrome, epilepsy, palatal myoclonus, and subacute cerebellar ataxia, DBN may represent a GAD-Ab associated neurological syndrome. We suggest that GAD-Ab should be tested in patients with idiopathic DBN.

\section{ACKNOWLEDGEMENTS}

The authors gratefully thank Dr P La Rosa, Department of Otorhinolaryngology of the University of Rome "La Sapienza" for his contribution.

\section{Authors' affiliations}

G Antonini, F Giubilei, F Gragnani, V Ceschin, S Morino, E Bucci,

N Accornero, Department of Neurological Sciences, University of

Rome, "La Sapienza", Rome, Italy

R Nemni, Fondazione Don C Gnocchi, University of Milan, Milan, Italy

Competing interests: none declared.

Correspondence to: $\operatorname{Dr} G$ Antonini, Dipartimento di Scienze neurologiche, Viale dell'Università 30, 00185 Rome, Italy; antoninimusc @uniromal.it

Received 29 July 2002

Accepted in revised form 27 November 2002

\section{REFERENCES}

1 Halmagyi GM, Rudge P, Gresty MA, et al. Downbeating nystagmus. A review of 62 cases. Arch Neurol 1983:40:777-84.

2 Meinck HM, Faber L, Morgenthaler N, et al. Antibodies against glutamic acid decarboxylase: prevalence in neurological diseases. J Neurol Neurosurg Psychiatry 2001;71:100-3.

3 Dinkel K, Meinck HM, Jury KM, et al. Inhibition of gamma-aminobutyric acid synthesis by glutamic acid decarboxylase autoantibodies in stiff-man syndrome. Ann Neurol 1998:44:194-201.

4 Solimena M, Folli F, Aparisi R, et al. Autoantoibodies to GABA-ergic neurons and pancreatic beta cells in stiff-man syndrome. N Engl J Med 1990;322:1555-60.

5 Honnorat J, Saiz A, Giometto B, et al. Cerebellar ataxia with anti-glutamic acid decarboxylase antibodies. Arch Neurol 2001;58:225-30

6 Peltola J, Kulmala P, Isojarvi J, et al. Autoantibodies to glutamic acid decarboxylase in patients with therapy-resistant epilepsy. Neurology 2000;55:46-50

7 Nemni R, Braghi S, Natali-Sora MG, et al. Autoantibodies to glutamic acid decarboxylase in palatal myoclonus and epilepsy. Ann Neurol 1994;36:665-7.

8 Mitoma H, Song SY, Ishida K, et al. Presynaptic impairment of cerebellar inhibitory synapses by an autoantibody to glutamate decarboxylase. J Neurol Sci 2000;175:40-4

9 Leigh RJ, Zee DS. The neurology of eye movements. 3rd edn. New York: Oxford University Press, 1999.

10 Bohmer A, Straumann D. Pathomechanism of down-beat nystagmus: a simple hypothesis. Neurosci Lett 1998;250:127-30.

11 Ito $M$, Nisimaru N, Yamamoto M. Specific patterns of neuronal connections involved in the control of the rabbit's vestibulo-ocular reflexes by the cerebellar flocculus. J Physiol 1977;265:833-54.

12 Zee DS, Yamazaki A, Butler PH, et al. Effects of ablation of flocculus and paraflocculus on eye movements in primate. J Neurophysiol 1981;46:878-99.

13 Babalian AL, Vidal PP. Floccular modulation of vestibuloocular pathways and cerebellum-related plasticity: an in vitro whole brain study. Neurophysiol 2000:84:2514-28.

$14 \mathrm{Kim}$ J, Namchuk M, Bugawan T, et al. Higher autoantibody levels and recognition of a linear $\mathrm{NH} 2$-terminal epitope in the autoantigen GAD-65 distinguish stiff-man syndrome from insulin-dependent diabetes mellitus. $J$ Exp Med 1994; 180:595-606.

15 Baekkeskov S, Aanstoot HJ, Christgau S, et al. Identification of a $64 \mathrm{~K}$ autoantigen in insulin-dependent diabetes as the GABA-synthesizing enzyme glutamic acid decarboxylase. Nature 1990;347:151-6. 